\title{
Higgs in fermionic channel (CMS)
}

\author{
Camille Beluffi ${ }^{1}$, a \\ ${ }^{1}$ Centre for Cosmology, Particle Physics and Phenomenology (CP3) \\ Universite catholique de Louvain \\ Chemin du Cyclotron, 2 \\ B-1348, Louvain-la-Neuve \\ Belgium
}

\begin{abstract}
Searches for the Higgs boson have been carried out in different fermionic decay modes with the CMS detector at the LHC collider. The analysis is based on pp collision data collected at centre-of-mass energies of 7 and $8 \mathrm{TeV}$ corresponding to integrated luminosities of about $5 \mathrm{fb}^{-1}$ and $20 \mathrm{fb}^{-1}$ respectively. The strategy and results of the searches are reported.
\end{abstract}

\section{Introduction}

The 4th of July 2012, the CMS and ATLAS collaborations announced the observation of a new particle, whose properties are compatible with a standard model (SM) Higgs boson [1][2][3]. Since then, precise measurements of these properties have been performed: the latest mass measurement is 125.03 $\pm 0.30 \mathrm{GeV}$, the signal strength $\sigma / \sigma_{S M}$ is compatible with 1 , and the couplings with bosons have been established ( $\gamma \gamma, \mathrm{ZZ}$ and WW)[4][5]. However, to claim that this newly observed particle is the SM Higgs boson, the couplings to the leptons have to be proved as well. The Higgs can be produced in several ways, mainly by gluon-gluon fusion ( $\mathrm{ggH}$ ), but also by Vector Boson Fusion (VBF), in association with a $\mathrm{W} / \mathrm{Z}$ boson $(\mathrm{VH})$, or in association with a top quark pair (ttH). It mostly decays into a pair of b-quarks (57\%), in a pair of two $\tau$ leptons (6.3\%), and more rare into a pair of muons $(0.02 \%)$. Combining the production and decay modes leads to 12 channels, all of them covered in CMS[6].

In this document, the Higgs boson decays to $\tau \tau$ is presented in section 2, followed by the decay mode $H \rightarrow b \bar{b}$ in section 3. The fermionic combination can be found in section 4 . Then, the results for the Higgs produced in association with a top quark pair are shown in section 5, as well as the ones for the Higgs decaying to first and second generation leptons in section 6. Finally, conclusions are presented in section 7 .

\section{Higgs boson decays to $\tau \tau$}

The Higgs boson decays to $\tau \tau$ is a very significant channel that allows to directly measure the couplings to the leptons. A search is performed using the full available dataset of $4.9 \mathrm{fb}^{-1}$ at $\sqrt{s}=7 \mathrm{TeV}$

ae-mail: camille.beluffi@cern.ch 

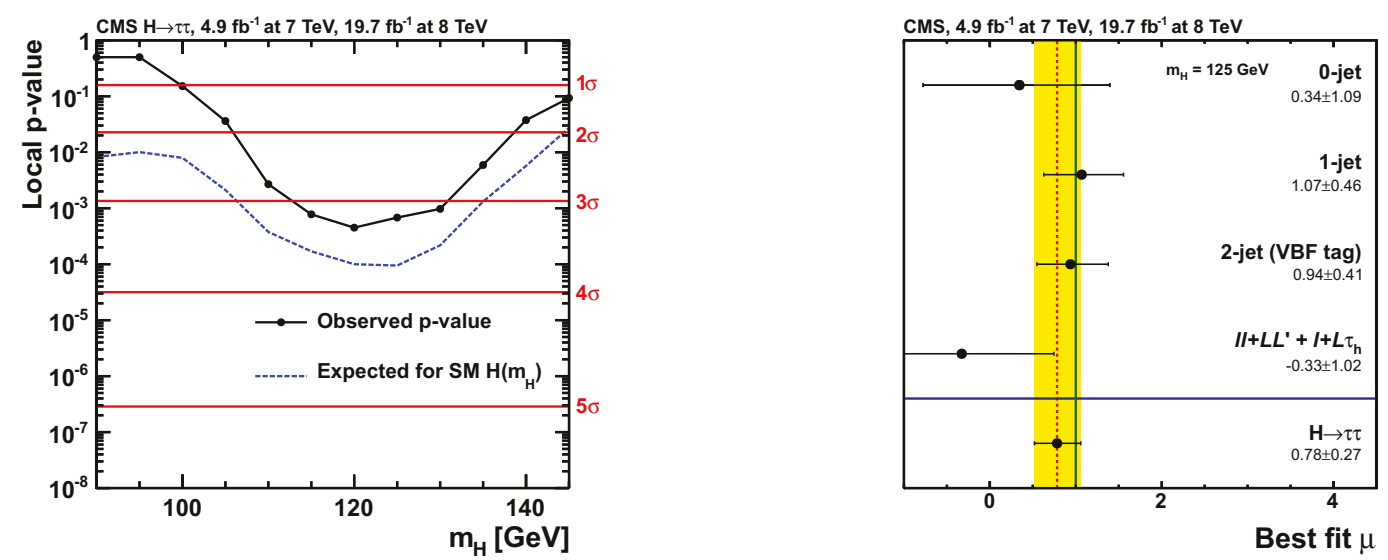

Figure 1: Left: local p-value and significance in number of standard deviations as a function of the SM Higgs boson mass hypothesis for the combination of all decay channels. Right: best-fit signal strength values, for independent categories, for $m_{H}=125 \mathrm{GeV}$.

and $19.7 \mathrm{fb}^{-1}$ at $\sqrt{s}=8 \mathrm{TeV}$ [7]. All the di-tau decay channels are used: $\mu \mu, e \mu, e e, \mu \tau_{h}\left(\tau_{h}\right.$ representing the hadronic decay of the $\tau$ ), $e \tau_{h}$ and $\tau_{h} \tau_{h}$. A categorization based on the number of jets and the boost of the system is applied, to constrain the backgrounds ( 0 -jet category), exploits boost of the Higgs system and the sensitivity to ggH production (1-jet category) and to obtain a high S/B, sensitive to the VBF production mode (2-jets category). To optimize further the signal selection, events are divided in categories based on the lepton $p_{T}$, di-tau $\mathrm{pT}$, and jet properties. The main backgrounds for this channel are the $Z \rightarrow \tau \tau, Z \rightarrow l l, \mathrm{~W}+$ jets events and QCD processes. Then, for most of the channels, the signal extraction is done with a likelihood fit on the di-tau mass. The VH production mode is also considered by requiring one or two additional electrons or muons compatible with $\mathrm{W}$ or $\mathrm{Z}$ boson decays. Eight additional channels are considered in this case, based on the decay products of the $\mathrm{W}$ or $\mathrm{Z}$ boson, and of the $\tau$ pair.

An excess of events over the background-only hypothesis is observed with a local significance of 3.2 standard deviations at $m_{H}=125 \mathrm{GeV}$, while the expected significance is 3.7 standard deviations and the best-fit value for the signal strength, $\mu=\sigma / \sigma_{S M}$, is $0.78 \pm 0.27$ times the SM prediction (see Fig.1), from which it can be conclude that there is evidence of the Higgs boson coupling to the $\tau$ lepton.

\section{Higgs boson decays to $b \bar{b}$}

Through the Higgs boson decays to $b \bar{b}$, the coupling between the Higgs and the down-type quark can be probed. Here, the ggH production is not directly considered because of the overwhelming QCD background. The signal topology of the production mechanism is exploited for the main production mode being the VH mode, and for the VBF mode, which is used separately to add some more sensitivity.

\subsection{VH mode}

The search is performed using the available $5.1 \mathrm{fb}^{-1}$ at $\sqrt{s}=7 \mathrm{TeV}$ and $18.9 \mathrm{fb}^{-1}$ at $\sqrt{s}=8 \mathrm{TeV}$ [8]. All the six channels for the leptonic decay of $\mathrm{V}$ are considered: $\mathrm{Z}(\mathrm{ll}) \mathrm{H}(\mathrm{bb})$ with $\mathrm{l}=\mathrm{e}, \mu, \mathrm{Z}(v v) \mathrm{H}(\mathrm{bb})$ and 

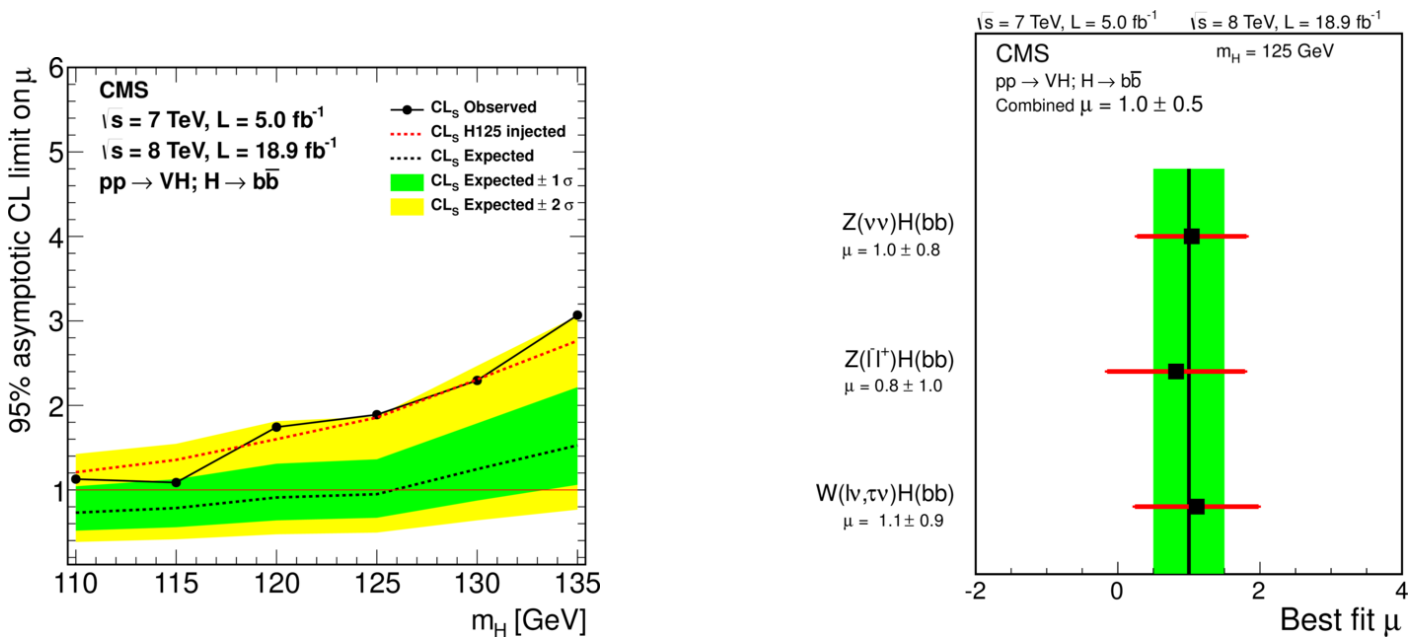

Figure 2: Left: the expected and observed 95\% CL upper limits on the product of the VH production cross section times the $\mathrm{H} \rightarrow b \bar{b}$ branching fraction, with respect to the expectations for the standard model Higgs boson. Right: the best-fit value of the production cross section for a $125 \mathrm{GeV}$ Higgs boson relative to signal strength, for partial combinations of channels and for all channels combined (band).

$\mathrm{W}(\mathrm{l} v) \mathrm{H}(\mathrm{bb})$ with $\mathrm{l}=\mathrm{e}, \mu, \tau$. Data control regions are used to normalize the main contributions $(\mathrm{V}+\mathrm{jets}, t \bar{t}$ and VV) To increase the background rejection, the resolution on the b-jet pair mass (jets arising from the hadronization of a b-quark) is improve by $15-25 \%$ by applying a Multi Variate Analysis (MVA) regression. The event categorization is based on the high boost ( $100 \mathrm{GeV}$ ) of the $\mathrm{V}$ bosons, with bins in $p_{T}(V)$, and multivariate discriminators are used for signal extraction in 14 regions defined.

An excess of events is observed with a local significance of 2.1 standard deviations at $m_{H}=125 \mathrm{GeV}$ (the expected significance is 2.1 standard deviations), and the best-fit value for the signal strength is $1.0 \pm 0.5$ times the SM prediction (see Fig.2), and therefore shows a very good agreement with SM expectations.

\subsection{VBF mode}

Here, the search is performed with the $19 \mathrm{fb}^{-1}$ recorded at $\sqrt{s}=8 \mathrm{TeV}$ [9]. This channel offers a very peculiar topology: 4 relatively hard jets, 2 central b-jets and 2 light quark jets with a large invariant mass $m_{q q}$ and large pseudo-rapidity separation $\Delta \eta_{q q}$. As a consequence, even with few signal events, it is possible to achieve a high signal over background ratio $(\mathrm{S} / \mathrm{B})$. A regression on the $m_{b \bar{b}}$ is also applied, and the event categorization is done in S/B bins using a multivariate discriminator. Finally the signal is extracted with a likelihood fit of the $m_{b \bar{b}}$ spectrum in each bin.

Upper limits on the cross section times the $H \rightarrow b \bar{b}$ branching ratio, in the VBF mode, are set. The observed (expected) limit is found to be 3.6 (3.0) times the SM prediction, and the best-fit value for the signal strength is $0.7 \pm 1.4$ times the SM prediction. 


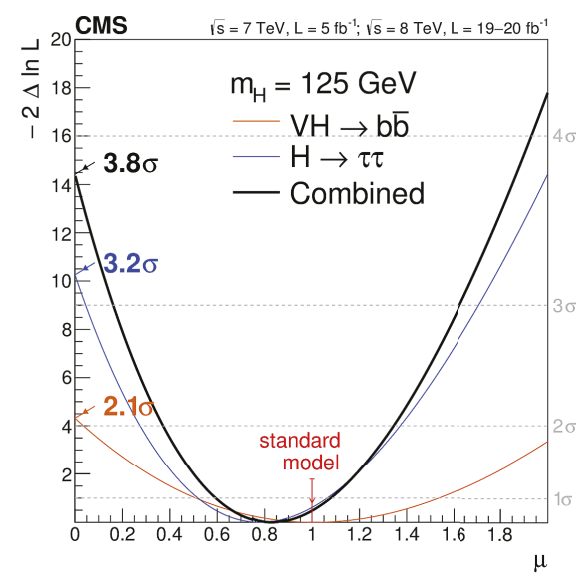

Figure 3: Scan of the profile likelihood as a function of the signal strength relative to the expectation for the production and decay of a standard model Higgs boson, $\mu$, for $m_{H}=125 \mathrm{GeV}$

\section{Fermionic combination}

A combination of the Higgs boson decays to $b \bar{b}$ channel (VH only) and Higgs boson decays to $\tau \tau$ is performed [10]. This combination results in strong evidence for the direct coupling of the $125 \mathrm{GeV}$ Higgs boson to fermions, with an observed (expected) significance of 3.8 (4.4) standard deviations (see Fig.3).

\section{Higgs boson produced in association with a top quark pair}

\section{$5.1 \mathrm{ttH}, H \rightarrow b \bar{b}:$ the standard method}

The Higgs boson production in association with a top quark pair is of main interest since it can be used to directly probe the Yukawa coupling between the Higgs and the top. This channel having a very low signal cross section, the decay modes $H \rightarrow \tau \tau$ and $H \rightarrow b \bar{b}$ are combined [11]. The dileptonic and semi-leptonic decay mode of $t \bar{t}$ are used, resulting in complex final states with large values of the number of jets and/or b-jets. It is used to create six categories to improve the signal over background ratio. The main irreducible backgrounds are the top pair production in association with heavy flavour jets $(\mathrm{tt}+\mathrm{HF})$ or in association with a $\mathrm{Z}$ boson. The signal/background discrimination is done with multivariate discriminators in each event category, using object kinematics, event shape and b-tagging information.

No significant excess of events is observed, and 95\% confidence level (CL) upper limits are set on the possible presence of a SM signal. The observed (expected) limit for $m_{H}=125 \mathrm{GeV}$ at $\sqrt{s}=8 \mathrm{TeV}$ is 5.2 (4.1) times the SM prediction (see Fig.4).

\section{2 ttH combination}

The mentioned fermionic ttH searches from the previous section are combined with $H \rightarrow \gamma \gamma$ and $\mathrm{H} \rightarrow$ Multi - leptons, with the same production mode [12]. The combined observed upper limit on the signal strength parameter $\mu$ is 4.3 for a Higgs with a mass at $125 \mathrm{GeV}$, while the expected is 1.8. The signal strength is $2.5_{-1.0}^{+1.1}$ (see Fig.5). 


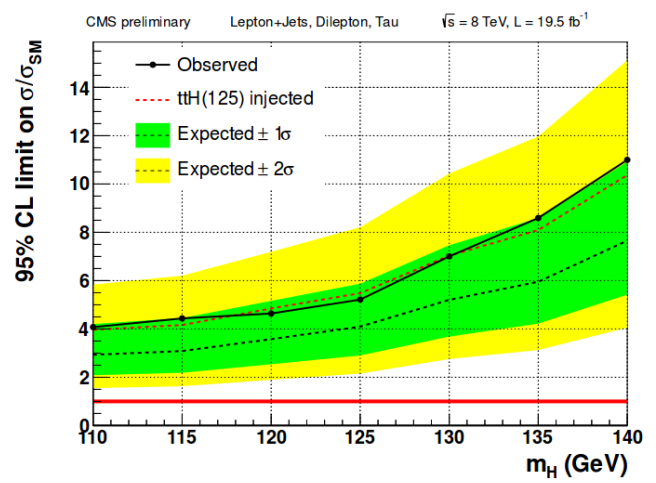

Figure 4: Using the $2011+2012$ datasets, the observed and expected 95\% CL upper limits on the signal strength parameter for $H \rightarrow \tau \tau$ and $H \rightarrow b \bar{b}$ combined.
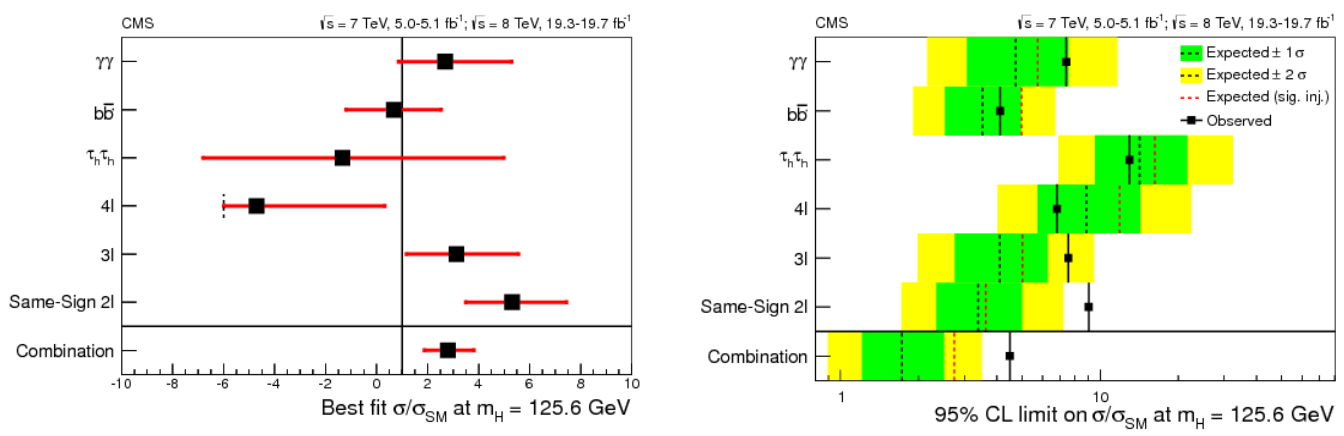

Figure 5: Left: best-fit values of the signal strength parameter for each ttH channel at $m_{H}=125 \mathrm{GeV}$. Right: 95\% CL upper limits on the signal strength parameter for each $\mathrm{ttH}$ channel at $m_{H}=125 \mathrm{GeV}$.

\section{3 ttH, $H \rightarrow b \bar{b}$, using the Matrix Element Method}

A new analysis is using the Matrix Element Method (MEM) to perform the search of the Higgs produced in association with a top quark pair and decaying into a pair of b-quark [13]. First, a likelihood ratio discriminant is created to distinguish the signal and the $\mathrm{tt}+\mathrm{HF}$ processes from the top pair production in association with light-flavour jets. Then, the MEM is applied to separate the signal from the $\mathrm{tt}+\mathrm{HF}$ events: it assigns to each event a weight under the signal and background hypothesis, and this weight is used to build the final discriminating variable, $P_{s / b}$, defined as the ratio between the two weights. To improve further the categorization, three categories are build in the semi-leptonic channel, based the $\mathrm{W}$ boson reconstruction.

Using the $19.5 \mathrm{fb}^{-1}$ available at $\sqrt{s}=8 \mathrm{TeV}$, the observed upper limit on the signal strength parameter $\mu$ is 3.3 for a Higgs with a mass at $125 \mathrm{GeV}$, while the expected limit is set at 2.9 (3.9 with signal injection, see Fig.6). The best-fit value for the signal strength is $0.7 \pm 1.4$. It should be mentioned that these results are $30 \%$ better than the ones using the standard approach, mainly because of the optimization of the signal and $\mathrm{t}+\mathrm{HF}$ separation. 


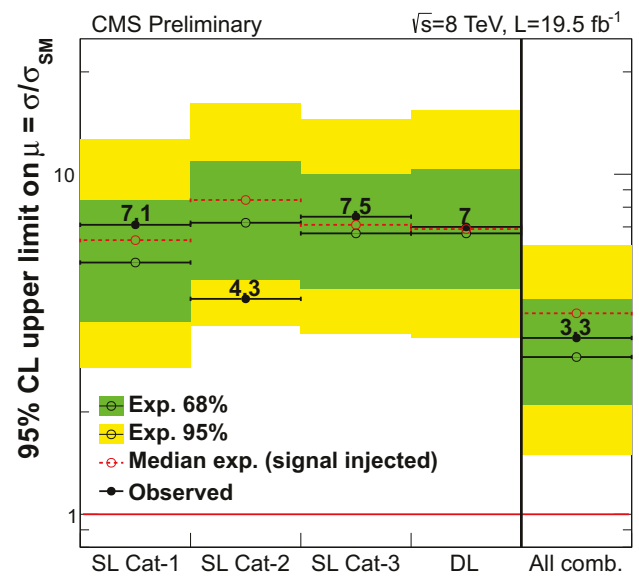

Figure 6: The 95\% CL upper limits on the signal strength parameter for each event category: the 3 categories in semi-leptonic channel and the dileptonic category.

\section{Higgs boson decays to first and second generation leptons}

\section{1 $H \rightarrow \mu \mu$ and $H \rightarrow e e$}

One of the properties that has to be checked is the couplings to first and second generation leptons. Indeed, the SM Higgs decays to fermions should not be universal. However the branching ratio for $H \rightarrow \mu \mu$ is very low (10 times smaller than $H \rightarrow \gamma \gamma$ ), and even smaller for $H \rightarrow e e$. But the search takes advantage of the clean signature in the detector and the excellent dimuon invariant mass resolution [14]. It is performed by directly combining the full $\sqrt{s}=7 \mathrm{TeV}$ and $\sqrt{s}=8 \mathrm{TeV}$ datasets $\left(5.0+19.7 \mathrm{fb}^{-1}\right)$, using the ggH and VBF production modes (for the $\mathrm{VH}$ and ttH production mode the branching ratio is too small). Events are categorized based on the number or reconstructed jets attempting to separate the gluon-gluon fusion and VBF production components. The signal is extracted by means of a fit to the $m_{\mu \mu}$ distribution using signal and background shapes.

Upper limits on the cross section times the $H \rightarrow \mu \mu$ branching ratio are set. The observed (expected) limit is found to be 7.4 (5.1) times the SM prediction, corresponding to an excess of events with a local significance of $2.8 \sigma$ (see Fig.7, left part).

For $H \rightarrow e e$, an upper limit of $0.038 \mathrm{pb}$ is set on the cross-section times branching ratio for $m_{H}=125$ $\mathrm{GeV}$ at $8 \mathrm{TeV}$ (see Fig.7, right part).

\section{$6.2 H \rightarrow \mu \tau$}

A first dedicated analysis is performed to search for Higgs decay with lepton flavour violation, $H \rightarrow$ $\mu \tau$, using the $19.7 \mathrm{fb}^{-1}$ data recorded at $\sqrt{s}=8 \mathrm{TeV}$ [15]. The considered tau decays are the hadronic $\tau_{h}$ and electron $\tau_{e}$ decays and the search is based on the same basic event selection and categorization as for the $H \rightarrow \tau \tau$ search (see Section 2). However, some differences in kinematics are noticed (harder muon pT spectrum, $\Delta \phi_{\mu \tau}$ ). Here, a collinear approximation is assumed to reconstruct the Higgs boson mass (see Fig.8). The observed (expected) limit is found to be $1.57 \%(0.75 \%)$ of the SM prediction, which is a large improvement from previous limits, and it corresponds to an excess of events with a local significance of $2.5 \sigma$. 

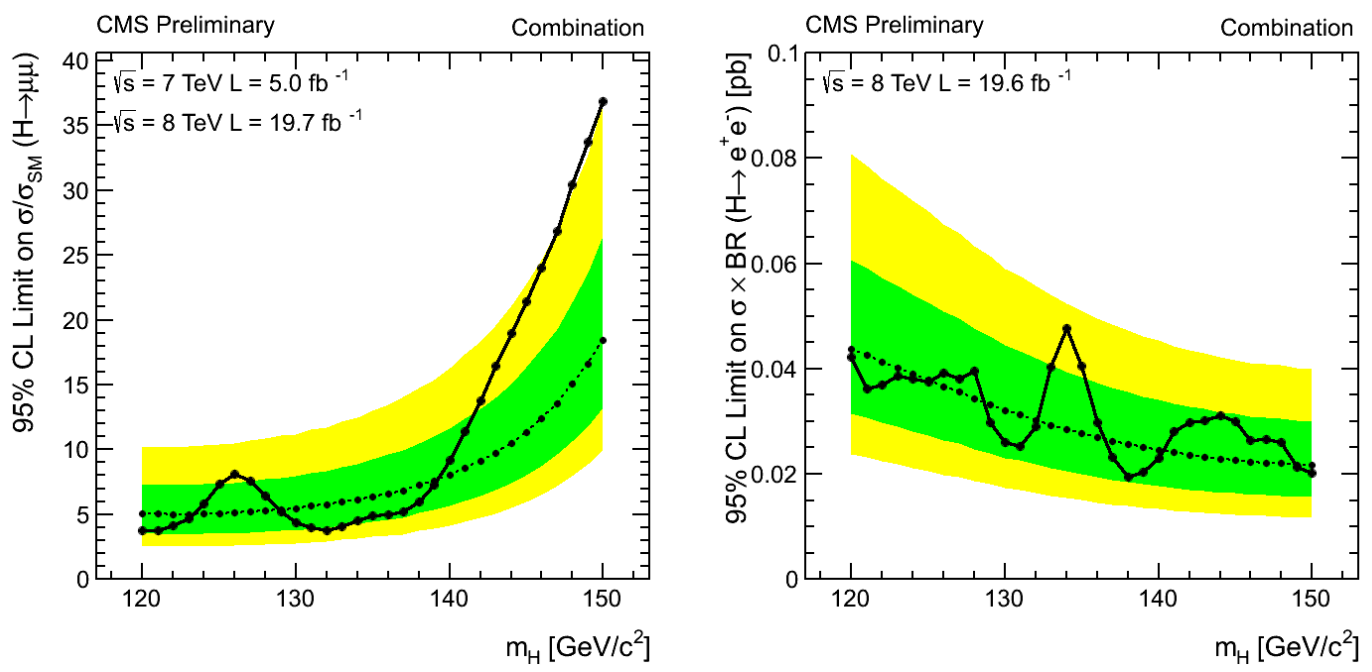

Figure 7: Left: mass scan for the expected and observed exclusion limits on $\sigma \times$ BR for $H \rightarrow \mu \mu$ for the combination at $\sqrt{s}=7$ and $8 \mathrm{TeV}$. Right: exclusion limits on $\sigma \times \mathrm{BR}$ for $\mathrm{H} \rightarrow \mathrm{e}^{+} \mathrm{e}^{-}$.

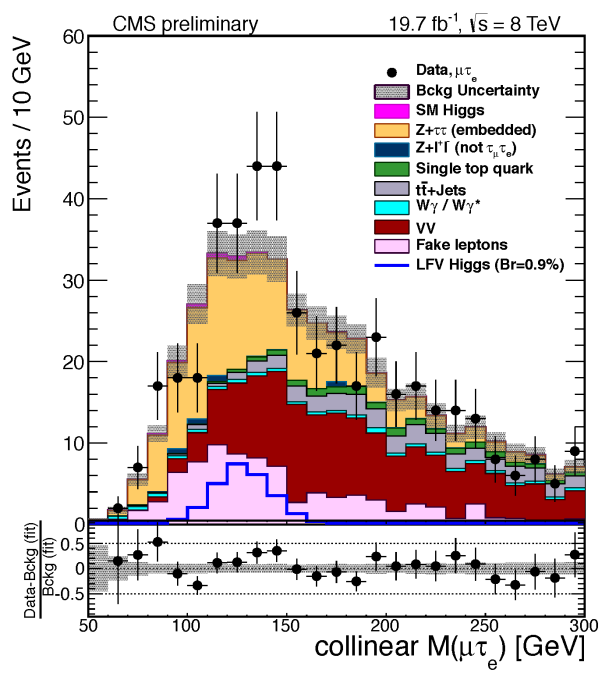

Figure 8: The collinear mass $M_{\text {collinear }}$ after fitting for signal and background for the LFV $H \rightarrow \mu \tau$ decays.

\section{Summary}

Searches for a SM Higgs boson decaying to fermions have been performed in CMS for different channels, $H \rightarrow \tau \tau, H \rightarrow b \bar{b}$ and $H \rightarrow \mu \mu$, using different production modes, and there is a strong evidence of the Higgs coupling to fermions. The excess seen for $H \rightarrow \tau \tau$ is of $3.2 \sigma$ and constitutes by itself an evidence, the excess in the $H \rightarrow b \bar{b}$ ( $\mathrm{VH}$ channel) is of $2.1 \sigma$, and combining these two 
channels, it leads to an evidence of $3.8 \sigma$ for the Higgs decaying to fermions. For the Higgs production in association with a top quark pair, the excess is at $2.7 \sigma$, when all the channels are combined. So far, the signal strength and the couplings measurements are consistent with the SM expectations, and searches as $H \rightarrow \mu \mu, H \rightarrow e e$ and $H \rightarrow \mu \tau$ should reveal them self interesting for the future.

\section{References}

[1] CMS Collaboration, "Observation of a new boson at a mass of $125 \mathrm{GeV}$ with the CMS experiment at the LHC", Phys. Lett.B716 (2012) 30.

[2] CMS Collaboration, "Observation of a new boson with mass near $125 \mathrm{GeV}$ in pp collisions at $\sqrt{s}=7$ and $8 \mathrm{TeV} "$, JHEP06 (2013) 081.

[3] ATLAS Collaboration, "Observation of a new particle in the search for the standard model Higgs boson with the ATLAS detector at the LHC", Phys. Lett. B716 (2012) 1.

[4] CMS Collaboration, "Measurement of Higgs boson production and properties in the WW decay channel with leptonic final states", JHEP01 (2014) 096.

[5] CMS Collaboration, "Measurement of the properties of a Higgs boson in the four-lepton final state", Phys. Rev. D89 (2014) 092007.

[6] CMS Collaboration, "The CMS experiment at the CERN LHC", JINST3 (2008) S08004.

[7] CMS Collaboration, "Evidence for the $125 \mathrm{GeV}$ Higgs boson decaying to a pair of tau leptons", JHEP05 (2014) 104.

[8] CMS Collaboration, "Search for the standard model Higgs boson produced in association with a W or a Z boson and decaying to bottom quarks", Phys. Rev. D89, 012003.

[9] CMS Collaboration, "Higgs to bb in the VBF channel", CMS-PAS-HIG-13-011.

[10] CMS Collaboration, "Evidence for the direct decay of the $125 \mathrm{GeV}$ Higgs boson to fermions", Nature Physics 10 (2014) (Advance online publication).

[11] CMS Collaboration, "Search for Higgs Boson Production in Association with a Top-Quark Pair and Decaying to Bottom Quarks or Tau Leptons", CMS PAS HIG-13-019.

[12] CMS Collaboration, "Search for the associated production of the Higgs boson with a top-quark pair", CMS-HIG-13-029.

[13] CMS Collaboration, "Search for ttH events in the $H \rightarrow b \bar{b}$ final state using the Matrix Element Method", CMS PAS HIG-14-010.

[14] CMS Collaboration, "Search for the standard model Higgs boson in the dimuon decay channel in pp collisions at $\sqrt{s}=7$ and $8 \mathrm{TeV}$ ", CMS-PAS-HIG-13-007.

[15] CMS Collaboration, "Search for lepton flavor violating decays of the Higgs boson", CMS PAS HIG-14-005. 